\title{
The development of curvature in the porcine radioulna
}

\author{
Jess Pantinople $^{1}{ }^{\text {, }}$ Kyle McCabe ${ }^{1}{ }^{\text {, }}$ Keith Henderson ${ }^{1}$, Hazel L Richards ${ }^{\text {Corresp., }}{ }^{1}$, Nick Milne ${ }^{1}$ \\ ${ }^{1}$ School of Anatomy, Physiology and Human Biology, University of Western Australia, Perth, Western Australia, Australia \\ Corresponding Author: Hazel L Richards \\ Email address: hazel.I.richards@gmail.com
}

Long bone curvature in animal limbs has long been a subject of interest and much work has explored why long bones should be curved. However, the 'when' and 'how' of curvature development is poorly understood. It has been shown that the rat tibia fails to attain its normal curvature if the action of muscles is removed early in life, but it is not clear if this is because the curvature fails to develop or if the bone becomes straighter without the action of muscles. No studies have examined the development of bone curvature in a normally developing quadruped, so this study tracks the course of curvature formation in the radioulna in a series of growing pigs. We also histologically examined the epiphyseal growth plates of these bones to determine if they contribute to the formation of curvature. In all three epiphyseal plates examined, the proliferative zone is thicker and more densely populated with chondrocytes on the cranial (convex) side than the caudal (concave) side. Frost's chondral modelling theory would suggest that the cranial side of the bone is under more compression than the caudal side, and we conclude that this is due to the action of triceps extending the elbow by pulling on the olecranon process. These results support the idea that bone curvature is an adaptation to habitual loading, where longitudinal loads acting on the curved bone cause bending strains that counter the bending resulting from the habitual muscle action. 


\section{The development of curvature in the porcine radioulna}

2 Jess Pantinople ${ }^{1}$, Kyle McCabe ${ }^{1}$, Keith Henderson ${ }^{1}$, Hazel L Richards ${ }^{1}$, Nick Milne ${ }^{1}$

$3{ }^{1}$ School of Anatomy, Physiology \& Human Biology, The University of Western Australia, 35

4 Stirling Highway, Crawley, WA, Australia

5 Corresponding author: Hazel L Richards ${ }^{1}$

6 Email: hazel.1.richards@gmail.com

7

8 


\section{Abstract}

10 Long bone curvature in animal limbs has long been a subject of interest and much work has

11 explored why long bones should be curved. However, the 'when' and 'how' of curvature

12 development is poorly understood. It has been shown that the rat tibia fails to attain its normal

13 curvature if the action of muscles is removed early in life, but it is not clear if this is because the

14 curvature fails to develop or if the bone becomes straighter without the action of muscles. No

15 studies have examined the development of bone curvature in a normally developing quadruped,

16 so this study tracks the course of curvature formation in the radioulna in a series of growing pigs.

17 We also histologically examined the epiphyseal growth plates of these bones to determine if they

18 contribute to the formation of curvature. In all three epiphyseal plates examined, the proliferative

19 zone is thicker and more densely populated with chondrocytes on the cranial (convex) side than

20 the caudal (concave) side. Frost's chondral modelling theory would suggest that the cranial side

21 of the bone is under more compression than the caudal side, and we conclude that this is due to

22 the action of triceps extending the elbow by pulling on the olecranon process. These results

23 support the idea that bone curvature is an adaptation to habitual loading, where longitudinal

24 loads acting on the curved bone cause bending strains that counter the bending resulting from the 25 habitual muscle action. 


\section{Introduction}

Long bone curvature in animal limbs has been a subject of interest because it would seem to weaken the bone under weight-bearing conditions - that is, theoretically, curved bones are more prone to failure than straight bones (Bertram and Biewener 1988). Many studies have used strain gauges in vivo to examine the strains experienced by curved bones during locomotion (Lanyon and Baggott 1976; Lanyon and Bourn 1979). More contemporary studies have used finite element analysis to model these strains under load (Jade et al. 2014; Milne 2016), while others have studied the relationship between body size and bone curvature (Biewener 1983; Swartz 1990; Shackelford and Trinkaus 2002). It is widely understood that altering the mechanical environment of bones affects their growth (Mosley 2000) and metabolism (Uhthoff \& Jaworski 1979) - for instance, Lanyon (1980) showed that rat tibiae failed to attain normal curvature if loads were removed by sciatic neurectomy and patella tenotomy.

In their study, Lanyon (1980) stated that the tibia 'fails to attain' its normal curvature, but did not clarify whether the bone was initially straight and did not develop its normal curvature, or if it was initially curved and became straighter due to the intervention. Searching the literature failed to reveal a clear account of when and how the curvature of such bones appears. Therefore, the first aim of this study is to document when bone curvature develops, using radioulnae from a series of fetal through to six-month-old pigs (Sus scrofa domesticus). If the curvature of the radioulnae increases as the young pig grows and begins to use its forelimbs in locomotion, the idea of curvature development as a response to locomotor loading will be supported.

The second question addressed here is how such curvature develops. There are two ways a bone may alter its shape or size: endochondral growth during development; and surface remodelling. Frost (1973) described how bones might adjust their shape by surface remodelling in response to loading. Indeed, a curved bone under longitudinal loading would be expected to become straighter, because the concave surface would be under compression - and thus depository - and the convex side would be under tension and thus resorptive. Such a bone would straighten by surface remodelling (drift) under these loads. Conversely, Frost described how an eccentrically loaded bone may become curved by the same mechanism (Frost 1964).

Developing bones have epiphyseal growth plates which are the principal means of bone elongation. Frost (1979) described a chondral modelling theory - he suggested that articular or 
59 epiphyseal growth cartilages respond to differences in loading: growing faster under

60 physiological compression and slower under tension. Asymmetric growth at the distal femoral

61 epiphysis has been shown to contribute to the orientation of the knee, producing the carrying

62 angle in humans (Tardieu and Trinkaus 1994). Cartilage can also form at the site of repair in

63 bone fractures, and it has been demonstrated, in newborn mice, that these chondrocytes respond

64 in the same way to tension and compression as those concerned with epiphyseal growth (Rot et

65 al. 2014). This is the mechanism by which these bones can straighten during fracture repair (Rot

66 et al. 2014). It is possible that such uneven chondrocyte activity, in response to compressive and

67 tensile loading, may be a way of producing curvature during development.

68 So, our second approach in this study is to examine the cranial and caudal sides of the epiphyseal

69 plates of the porcine radius and ulna, and measure the thickness of the proliferative (growth) zone as well as the density of chondrocytes within this zone. This zone is identifiable as the area where chondrocytes appear flattened and are arranged in columns (Niehoff et al. 2004). If the growing bone is increasing in curvature, it is expected that the proliferative zone on the side of concave side. 


\section{Materials \& Methods}

78 Twenty-two Duroc boar $\times$ Landrace $\times$ Large White crossbred pigs which died in the normal 79 course of farming operations were collected from a pig farm in Western Australia (UWA Animal 80 Ethics Committee approval F 69199). The pigs ranged in age from eight days prenatal to 23 81 weeks postnatal. The ages of the postnatal pigs were obtained from the supplier whereas those 82 of the prenatal pigs were derived by measuring crown-rump length (Ullrey et al 1965). One 83 additional 72-week-old pig was also used to compare the developing pigs with a mature adult. 84 Each pig was weighed, the right radioulna removed and cleaned, and the bone length measured 85 (Table 1). The radioulnae were photographed from the lateral side using a standardised 86 orientation, ensuring the axis of the trochlea notch was vertical in each photograph. From the 87 photographs, 16 landmarks and 80 semilandmarks were digitised in tpsDig (Rohlf 2016). The landmarks were chosen to describe the overall shape of the bone, and the semilandmarks were used to characterise the curvature of the bones' caudal and cranial surfaces in the sagittal plane 90 (see Fig 1 and Table 2).

91 The coordinates of the three sets of semilandmarks, describing the curvature of the posterior ulna 92 and the anterior and posterior radial margins, were translated, scaled and rotated so the proximal semilandmark was at the origin $(0,0)$ and the distal semilandmark had the coordinate $(1,0)($ after Richmond and Whalen 2001). The largest Y-value among the semilandmarks represented the normalised curvature (i.e. maximum subtense/chord length). 
97 Table 1. The age and weight of the pigs and the lengths of the radioulnae used.

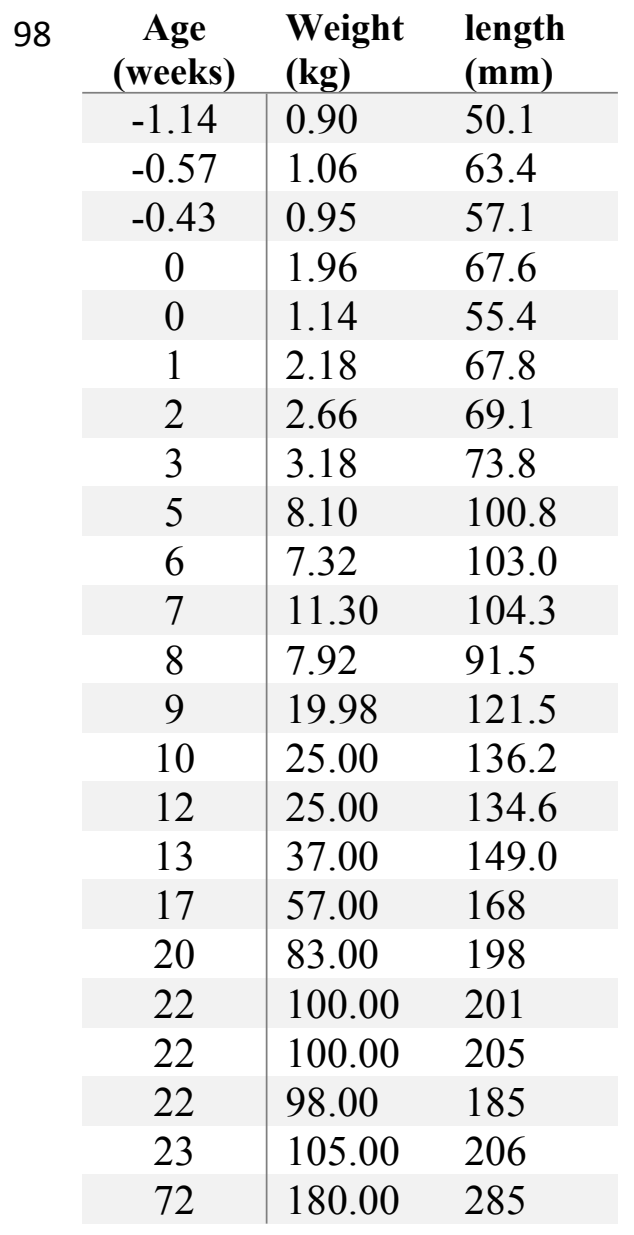

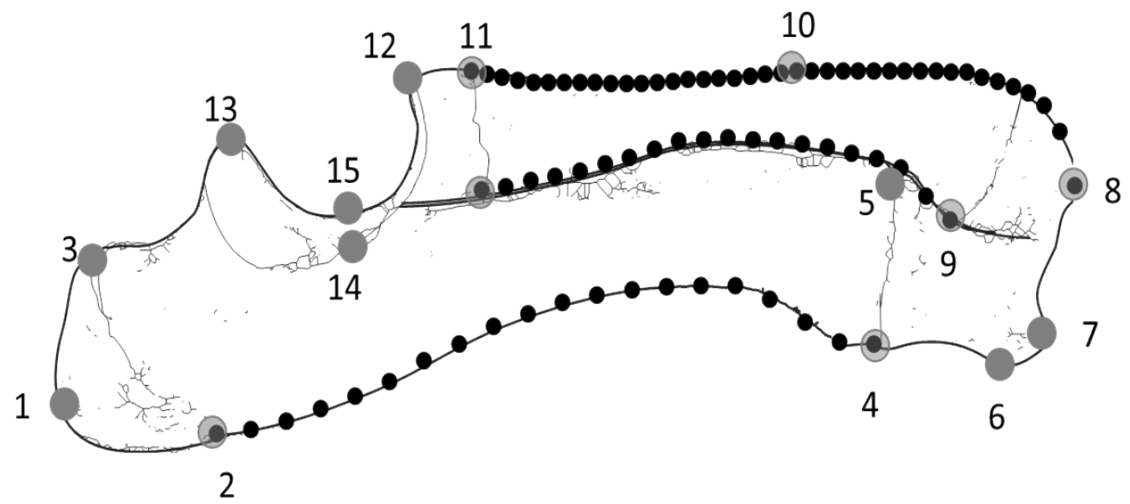

Figure 1. Diagram of a lateral view of the radioulna with the landmarks (numbered grey dots) and semilandmarks (black dots) used: $20 \mathrm{x}$ posterior ulna, $20 \mathrm{x}$ posterior radius and $40 \mathrm{x}$ anterior ulna. 
Table 2. The landmarks on the radioulna.

99

100

101

102

No. Definition

103

$1 \quad$ Apex of olecranon

2 Proximal epiphyseal plate at posterior margin of ulna

103

104

3 Proximal epiphyseal plate at anterior margin of ulna

105

106

4

Distal epiphyseal plate at posterior margin of ulna

5 Distal epiphyseal plate at anterior margin of ulna

6

Posterior-most point of distal surface of ulna

$7 \quad$ Styloid process of ulna

$8 \quad$ Styloid process of radius

9 Distal epiphyseal plate at posterior margin of radius

10 Middle point of anterior margin of radius

11 Proximal epiphyseal plate at anterior margin of radius

12 Proximal epiphyseal plate at posterior margin of radius (lateral)

13 Midpoint of distal trochlear surface

14 Lateral point of mid-trochlear surface

15 Midpoint of mid-trochlear surface

16 Midpoint of proximal trochlear surface 
107 The 96 landmarks were submitted to geometric morphometric analysis in morphologika

108 (O’Higgins and Jones 1998). First the landmark sets were Procrustes registered so differences in

109

110

111

112

113

114

115

116

117

118

119

120

121

122

123

124

125

126

127

128

129

130

131

132

133

134

size, position and orientation among the landmarks were removed and only differences in the shape described by the landmarks remained. The principal components analysis was applied to identify the main patterns of shape variation. As we were interested in the changes in shape that accompany growth, form space was used so that the first principal component (PC1) characterised the shape change with increasing size.

Six specimens of different ages (one week prenatal and one, five, nine, 17 and 22 weeks postnatal) were selected and their epiphyseal growth plates examined. The bones were sectioned in the sagittal plane, and samples of the distal ulnar, and proximal and distal radial epiphyseal plates were removed for histological analysis. The proximal ulnar plate was not included because it is a traction epiphysis for the olecranon process and does not form part of the shaft of the ulna (Morgan et al. 2000).

Each growth plate sample included the cranial and caudal sides of the epiphysis. The samples were fixed in 10\% neutral buffered formalin, rinsed in $0.9 \%$ saline and then immersed in rapid decalcifier (Apex Engineering Products Corp., Aurora, IL, USA) for between two and four hours. When the samples were soft enough, they were rinsed in saline and agitated in phosphatebuffered saline (PBS) for 15 minutes, then placed in 30\% sucrose solution made in PBS overnight. Samples were then agitated for 15 minutes in a 50:50 solution of PBS and OCT (optimal cutting temperature compound - ProSciTech, Thuringowa, QLD. Australia) before being embedded in OCT, frozen, and sectioned at $40 \mu \mathrm{m}$ using a cryostat at $-20^{\circ} \mathrm{C}$ (Leica CM 3050s). Each block had 25 sections cut and every fifth section was used. The sections were placed on Superfrost Plus slides (Lomb Scientific Pty Ltd., Taren Point, NSW, Australia) and air dried overnight. Initially the slides were rinsed and stained in the normal vertical orientation, but this resulted in the loss of some sections (so only four of the six distal radial sections were available). To avoid section loss, subsequent slides were laid horizontally, drops of tap water were used to wash off the OCT, and Gill's Haematoxylin I was applied for one minute before rinsing and mounting with Kaiser's aqueous mounting medium. 
135 Images of the epiphyseal plates were captured at 100× magnification (Olympus BX50

136 microscope). The images were exported to tpsDig, and the thickness of the proliferative zone

137 was measured in five locations $100 \mu \mathrm{m}$ apart, and the mean recorded (Fig 2).

138 Chondrocytes in the proliferative zone were counted using the optical fractionator probe in

139 Stereo Investigator (MBF Bioscience, Williston, VT, USA). This probe is used to directly count

140 proliferating chondrocytes within a defined volume, which then produces an unbiased population

141 estimate without being affected by the size, distribution and orientation of the chondrocytes

142 within this zone (West, Slomianka \& Gundersen 1991). The user identifies the region of interest

143 (proliferative zone) in five sections of each tissue block and Stereo Investigator randomly selects

14420 equidistant sampling sites in each section, so 100 sampling sites are counted for each tissue

145 block (Fig 2). From these estimates of chondrocyte population, cell density was estimated by

146 dividing cell count by the total volume of the sampling sites/optical disectors (West, Slomianka

$147 \&$ Gundersen 1991).

148 Using the statistical package Genstat (18 $8^{\text {th }}$ edition, VSN International), Shapiro-Wilks tests were 149 used to confirm normality of data. We then conducted linear regression to determine if the 150 curvatures of the posterior ulna, posterior radius and anterior radius increased significantly with 151 age. Paired t-tests were used to check for differences in the proliferative zone thickness and cell 152 density between the cranial and caudal side of each epiphyseal growth plate. 




Figure 2. A: An epiphyseal plate with the proliferative zone outlined in dashed lines. Counting frames (green and red boxes) were placed equidistantly across the proliferative zone. The black lines represent the measured thickness of the proliferative zone. B: Inset at higher magnification, showing which cell nuclei (yellow dots) in each counting frame were counted by the software (those wholly within the frame and those intersecting the green boundary). 
163

164

165

166

167

168

169

170

171

172

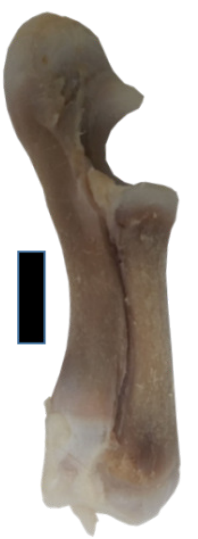

Neonate


23 weeks

173

Figure 3. PC1 and age. Form space PC1 $(98.5 \%)$ is plotted against age. Wireframe diagrams represent the shapes at the extremes of PC1 $(-1 \&+1)$, accompanied by representative photographs of neonatal and 23-week-old radioulnae (scale bars indicate $1 \mathrm{~cm}$ ). 
176 When the curvature measurements for the posterior ulna and the posterior and anterior margins

177 of the radius are regressed against age, all three curves increase. This increase is only significant

178 for the posterior ulna and anterior radial curves (Fig 4). The curvature value for the 72-week-old

179 pig is also indicated, and shows that the adult curvature is attained in these pigs by 23 weeks of

180 age. Indeed, the anterior radial curve diminishes somewhat in the older pig.

181 The mean thicknesses of the cranial and caudal sides of the epiphyseal proliferative zones are

182 shown in Fig 5. Paired t-tests show that the cranial side is significantly thicker than the caudal

183 side in all three growth cartilages examined $(\mathrm{P}<0.01)$. Figure 6 shows the chondrocyte density,

184 and again paired t-tests show these cells are more densely packed on the cranial than the caudal

185 side $(\mathrm{P}<0.01)$. Photomicrographs of the cranial and caudal sides of the three cartilage growth

186 plates are shown in fig 7. In each case the cranial side is seen to have a greater chondrocyte

187 density than the caudal side.
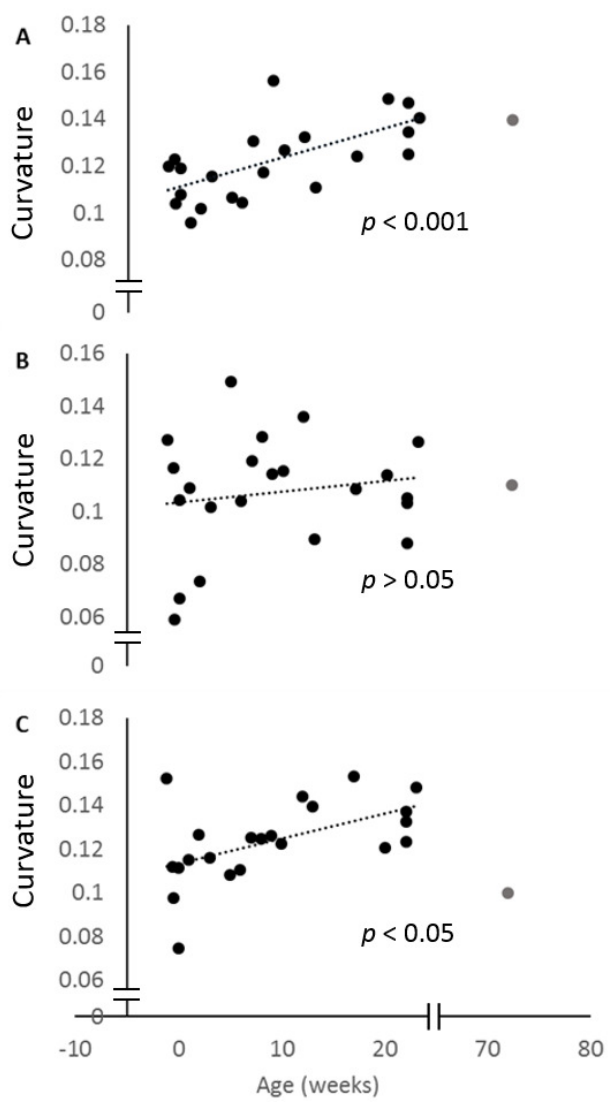

Figure 4. Change in curvature with age. Plots of the normalised curvature versus age for $\mathrm{A}$ ) posterior ulna, B) posterior radius and C) anterior radius. The values for the 72 -weekold pig (grey circle) are included to show how curvatures change beyond 23 weeks, but this specimen was not included in the regression analyses. The $p$ values show the significance of the regression analyses. 

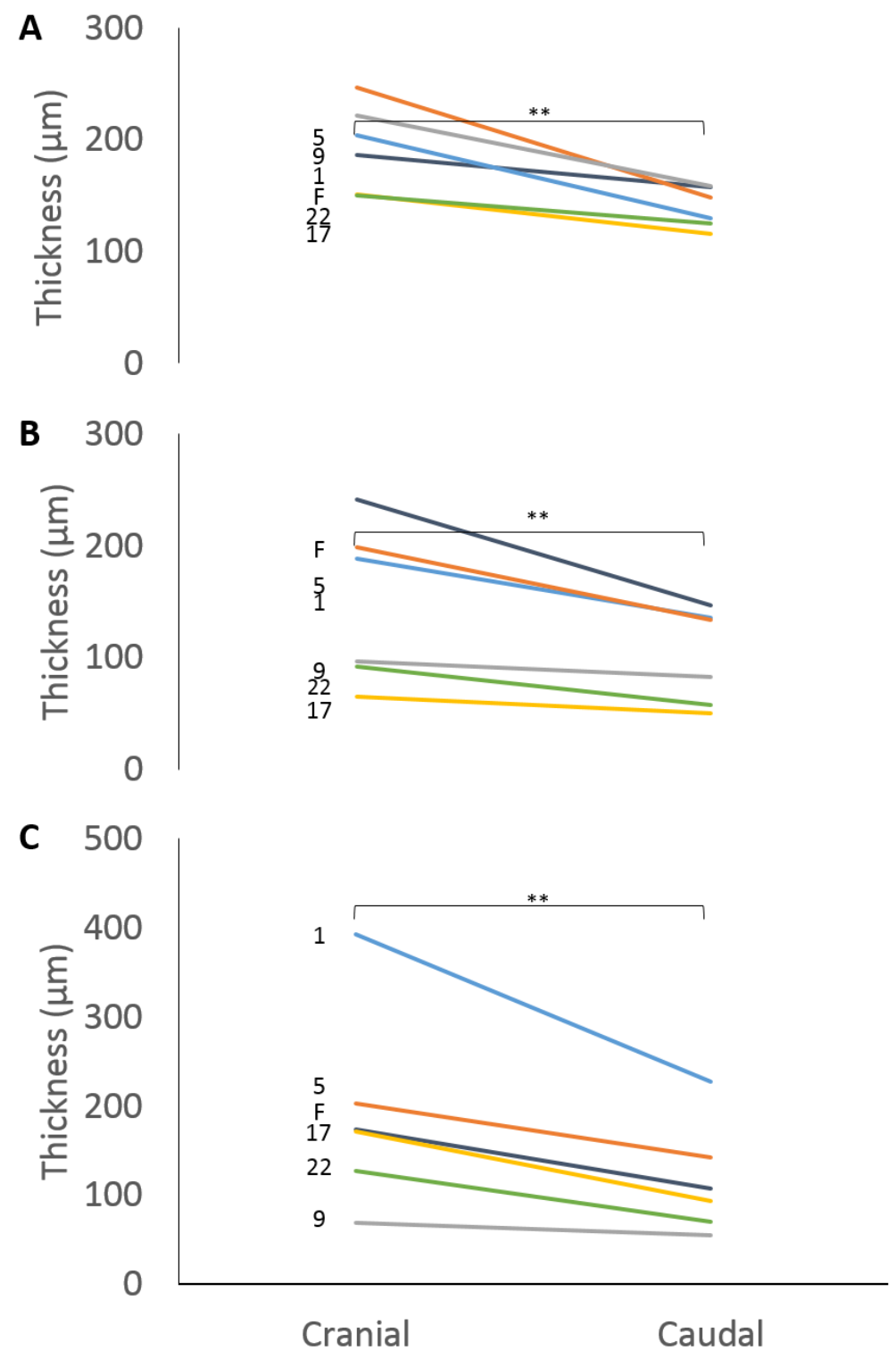

Figure 5. Thickness of the cranial and caudal regions of the proliferative zone in A) distal ulnar, B) proximal radial and C) distal radial epiphyseal growth plates. Individual lines represent a single specimen with age (in weeks) indicated. **Paired t-tests show that that the cranial is significantly thicker than the caudal side $(p<0.01)$. 
190

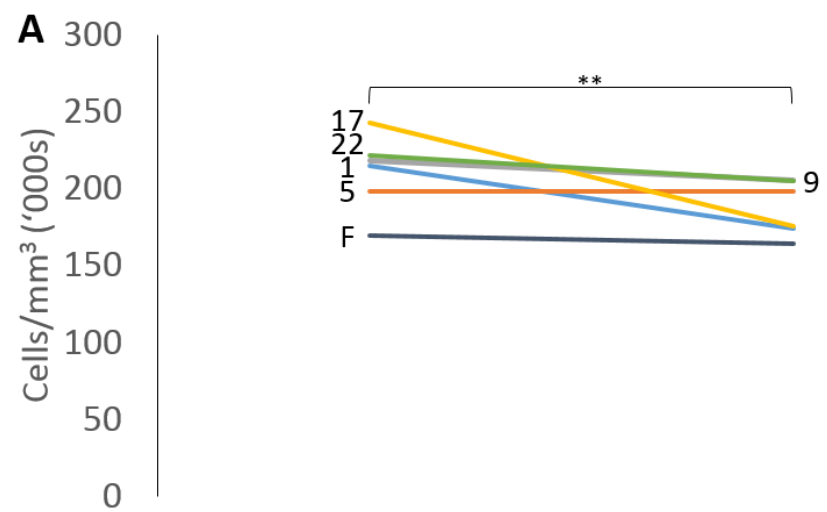



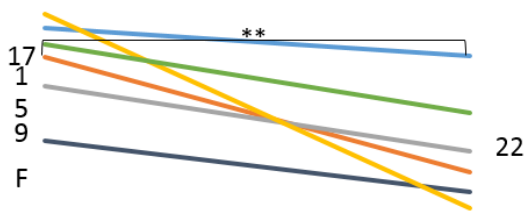

C 300

$\underbrace{250} 200$

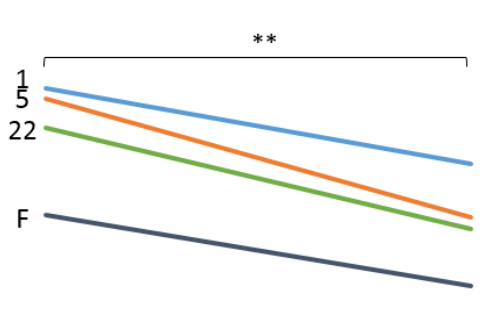

50

0

Cranial

Caudal

Figure 6. Chondrocyte density in the cranial and caudal regions of the proliferative zone in A) distal ulnar, B) proximal radial and C) distal radial epiphyseal growth plates. Individual lines represent a single specimen with age (in weeks) indicated. **Paired t-tests show that that the cranial has a significantly denser chondrocyte population than the caudal side $(p<0.01)$. 
Cranial

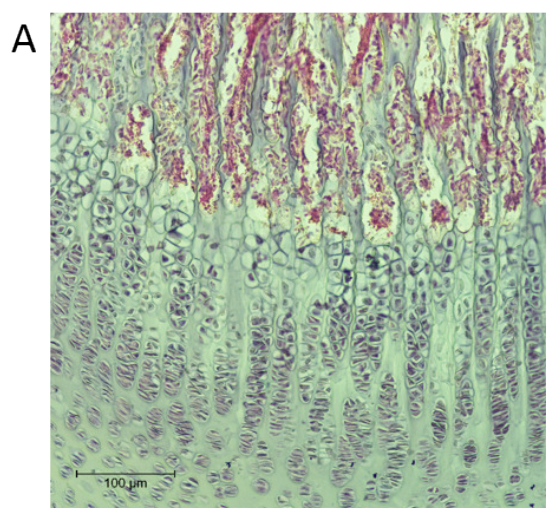

C

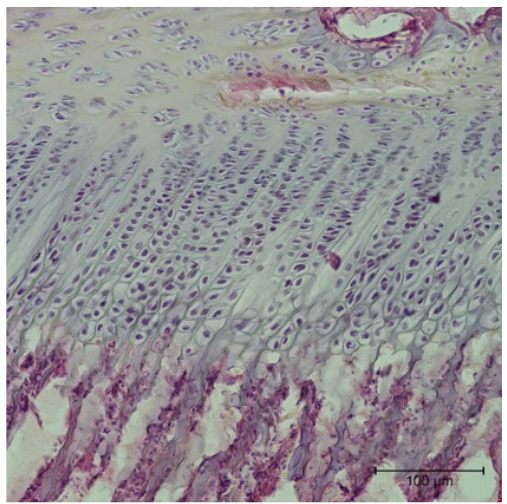

$\mathrm{E}$

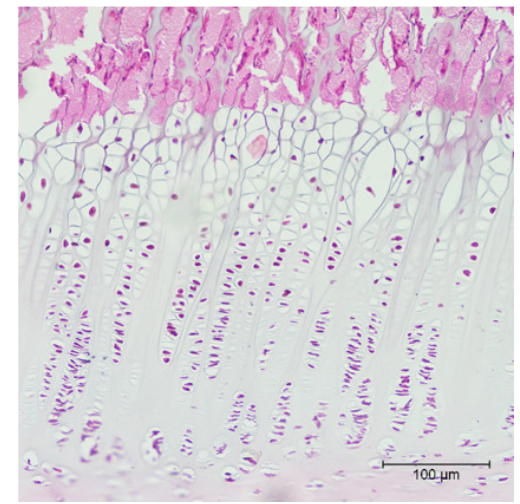

Caudal
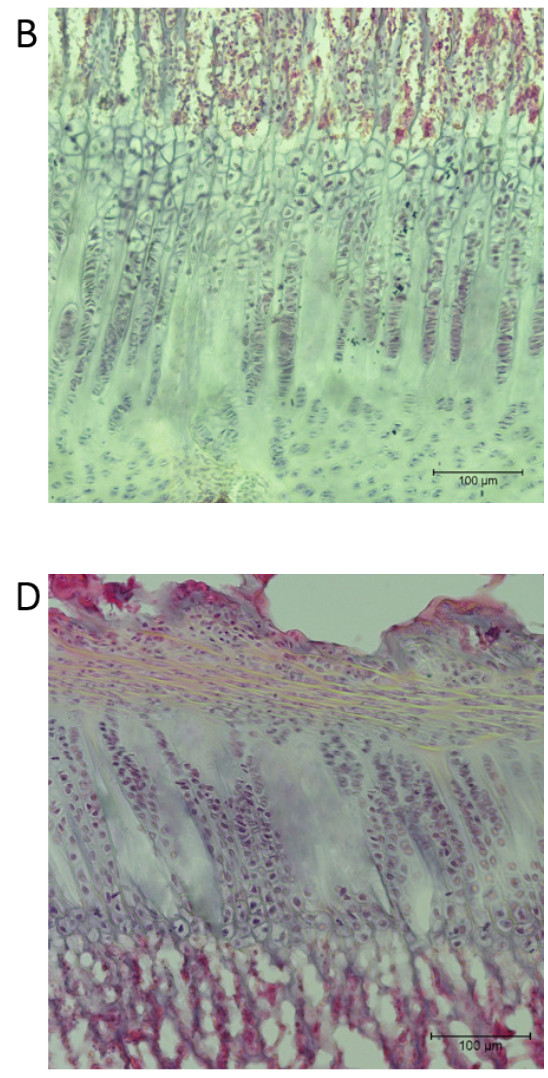

$\mathrm{F}$

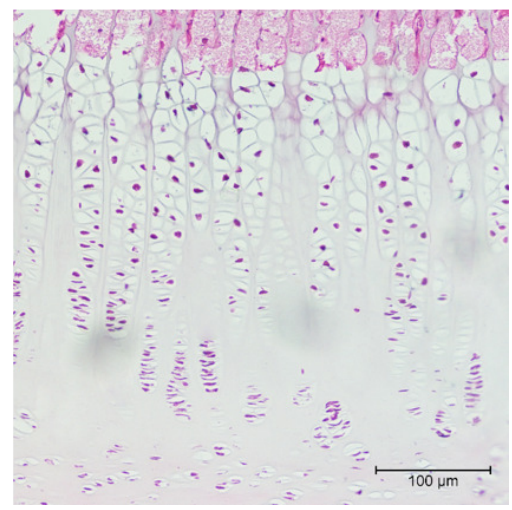

Figure 7. Photomicrographs showing examples of the cranial and caudal growth cartilages from the distal ulna (A and B), proximal radius (C and D) and distal radius (E and F). Sections $\mathrm{A}$ to $\mathrm{D}$ are $40 \mu \mathrm{m}$ thick and stained with haematoxylin. Sections $\mathrm{E}$ and $\mathrm{F}$ are $8 \mu \mathrm{m}$ thick and strained with haematoxylin and eosin. All sections are presented at the same magnification (see $100 \mu \mathrm{m}$ scale bar). 
195

196

197

198

199

200

201

202

203

204

205

206

207

208

209

210

211

212

213

214

215

216

217

218

219

220

221

222

223

\section{Discussion}

This study explored the 'when' and the 'how' of curvature development in the pig radioulna. Both the radius and ulna were found to be caudally curved in near-term fetuses, and this curvature was shown to increase in the first six months of life. Examination of the epiphyseal growth plates showed more chondrocyte activity in the cranial (convex) side than the caudal (concave) side. This establishes a mechanism for the formation and maintenance of bone curvature during development. The differential growth in the cranial and caudal sides of the epiphysis may occur in response to bending strains that place the cranial side in compression and the caudal side in tension (or less compression) (Frost 1964, Milne 2016).

The geometric morphometric analyses and regressions of curvature against age showed that radioulnar curvature increased between two weeks prenatal and 23 weeks postnatal. This was true for both the entire bone, as seen in the GM analyses, and for the posterior ulnar and anterior radial margins individually. However, the posterior margin of the radius did not significantly increase in curvature over this period. The slope of the posterior radial curve regression line was shallower, and the variation among curve values greater, than in the other measured curvatures (see Fig 4). It may be that the contact between the posterior ulna and the anterior radius impedes curvature development there, or that clear identification of the anterior surface of the radius was difficult and affected our measurements.

Here we examined pigs between two weeks prenatal and 23 weeks postnatal. The prenatal pigs already had some curvature in their radioulnae so it is still uncertain whether this bone is curved throughout fetal life, or is straight during earlier stages of gestation. The initial fetal curvature seen here may be caused by primitive muscle actions in utero, causing forelimb movements.

Such movements are at their greatest frequency around 15 days before birth (Cohen et al. 2010). Examination of younger fetuses would determine whether the radioulna is indeed curved during early gestation, or if it begins as a straight bone prior to any muscle loading. It is also uncertain whether curvature changes any further beyond 23 weeks, but the 72-week-old specimen suggests that adult curvature is not greater than that of a 23-week-old. The geometric morphometric analysis (Fig 2) and the linear regressions of curvature on age show that the 23-week-old pigs had already attained or even overshot the mature adult curvature. 
224 Our analysis of the proliferative zone activity of the epiphyseal growth plates showed that this

225 zone is thicker and more densely populated with chondrocytes on the cranial than the caudal

226 side. In 1979, Frost proposed the chondral modelling theory - within the limits of physiological

227 loading, cartilage grows faster under compression than tension, and grows slower under less

228 compression than more. Numerous studies have supported this theory (e.g. Tardieu and Trinkaus

229 1994; Urban 1994; Hamrick 1999; Congdon et al 2012; Rot et al 2014). Asymmetrical growth of

230 the epiphyseal plates can influence bone shape and was purported to be the mechanism behind

231 both the normal formation of the carrying angle in human femora (Shackelford and Trinkaus

232 2002) and the straightening of bones as fractures heal (Rot et al. 2014). Formation of bone

233 curvature may also be attributable to such asymmetrical epiphyseal plate growth, as our results

234 here suggest.

235 Recently, Henderson and colleagues (2017) showed that the ulnae of terrestrial primates and 236 marsupials are caudally curved, like that of the pig and other terrestrial quadrupeds. Conversely,

237 the ulna of arboreal primates and marsupials is cranially curved. Terrestrial quadrupeds' forearm

238 is dominated by the action of the triceps muscle acting to maintain extension of the elbow, but

239 arboreal species rely on the action of the brachialis muscle to flex the elbow and allow the

240 animal to climb and cling among the branches. The actions of triceps and brachialis place

241 opposite bending strains upon the ulna, and it is proposed that the corresponding opposite

242 curvatures are adaptations to those different bending strains (Milne 2016). If bone and cartilage

243 are stimulated to grow by compressive loading and growth is inhibited by tension (Frost 1979),

244 then this would provide a mechanism by which these opposite curvatures develop in terrestrial

245 and arboreal species. In the case of the developing pig, where triceps acts to maintain stance

246 during locomotion, the radioulna would experience cranial bending (with more compression on

247 the cranial side). These bending strains may be responsible for the greater proliferation of

248 cartilage cells in the cranial than the caudal side of the growth plates, and thus may be

249 responsible for the development of the caudal curvature (caudal concavity) observed here.

250

251 Conclusions

252 Until recently, the timing and the mechanism of bone curvature formation was unknown. Lanyon

253 (1980) saw a straightening of the rat tibia in the absence of muscle action but did not specify 
254 whether the bone curvature decreased or failed to form in the first place. The present study is the

255 first to document that curvature does exist early in life, and increases as the animal grows.

256 Frost's (1979) chondral modelling theory was used to explain how congruent joint surfaces are

257 maintained and how the femoral carrying angle forms - however, until now this mechanism has

258 not been explicitly associated with curvature formation. The results presented here suggest that

259 chondral modelling is also applicable to the formation of bone curvature. Further, it seems likely

260 that the formation of radioulnar curvature may be a direct result of the habitual action of the

261 triceps muscle, inducing a strain gradient that drives chondral modelling in the epiphyseal

262 growth plates of the growing bone. This was a pilot study exploring the development of

263 curvature and a possible mechanism of curvature formation; it is hoped that future studies will

264 test these ideas and further explore the mechanism of long bone curvature formation.

265

266 Acknowledgements

267 John Kopriwa provided the specimens used in the study. Lutz Slomianka, Mary Lee and Guy

268 Ben-Ary assisted in the preparation and analysis of the cartilage samples. 
270

271

272

273

274

275

276

277

278

279

280

281

282

283

284

285

286

287

288

289

290

291

292

293

294

295

296

297

298

299

\section{References}

Biewener, AA 1983, 'Allometry of quadrupedal locomotion: the scaling of duty factor, bone curvature and limb orientation to body size', Journal of Experimental Biology, vol. 105, pp. 147-171.

Cohen, S, Mulder, EJH, van Oord, HA, Jonker, FH, Parvizi, N, van der Weijden, GC \& Taverne, MAM 2010, 'Fetal movements during late gestation in the pig: A longitudinal ultrasonographic study', Theriogenology, vol. 74, no. 1, pp. 24-30.

Congdon, KA, Hammond, AS \& Ravosa, MJ 2012, 'Differential limb loading in miniature pigs (Sus scrofa domesticus): a test of chondral modeling theory', The Journal of Experimental Biology, vol. 215, no. 9, pp. 1472-1483. Available from: PMC.

Frost, HM 1964, The Laws of Bone Structure, Charles C Thomas, Springfield, IL.

Frost, HM 1973, Bone modelling and skeletal modelling errors, Charles C. Thomas, Springfield, IL.

Frost, H 1979, 'A chondral modeling theory', Calcified Tissue International, vol. 28, no. 1, pp. 181-200.

Hamrick, MW 1999, 'A Chondral Modeling Theory Revisited', Journal of Theoretical Biology, vol. 201, no. 3, pp. 201-208.

Henderson, K, Pantinople, J, McCabe, K, Richards, H \& Milne, N 2017 'Forelimb bone curvature in terrestrial; and arboreal mammals', PeerJ, 5, p:e3229

Jade, S, Tamvada, KH, Strait, DS \& Grosse, IR 2014, 'Finite element analysis of a femur to deconstruct the paradox of bone curvature', Journal of Theoretical Biology, vol. 341, pp. 53-63.

Lanyon, LE \& Baggott, DG 1976, 'Mechanical function as an influence on the structure and form of bone', The Journal of bone and joint surgery. British volume, vol. 58-B, no. 4, p. 436443.

Lanyon, LE \& Bourn, S 1979, 'The influence of mechanical function on the development of remodeling of the tibia' The Journal of bone and joint surgery. British volume, vol. 61-A pp263-272.

Lanyon, LE 1980, 'The influence of function on the development of bone curvature. An experimental study on the rat tibia', Journal of Zoology, vol. 192, no. 4, pp. 457-466. 
300

301

302

303

304

305

306

307

308

309

310

311

312

313

314

315

316

317

318

319

320

321

322

323

324

325

326

327

328

329

330

Milne, N 2016, 'Curved bones: An adaptation to habitual loading', Journal of Theoretical Biology, vol. 407, pp. 18-24.

Morgan, JP, Wind, AP \& Davidson, AP 2000, Hereditary bone and joint diseases in the dog: osteochondroses, hip dysplasia, elbow dysplasia, Schlütersche, Hanover.

Mosley, JR 2000, 'Osteoporosis and bone functional adaptation: Mechanobiological regulation of bone architecture in growing and adult bone, a review', Journal of Rehabilitation Research and Development, vol. 37, no. 2, pp. 189-99. Available from: ProQuest Central. Niehoff, A, Kersting, UG, Zaucke, F, Morlock, MM \& Brüggemann, G-P 2004, 'Adaptation of mechanical, morphological, and biochemical properties of the rat growth plate to dosedependent voluntary exercise', Bone, vol. 35, no. 4, pp. 899-908.

O'Higgins, P \& Jones, N 1998, 'Facial growth in Cercocebus torquatus: an application of threedimensional geometric morphometric techniques to the study of morphological variation', Journal of Anatomy, vol. 193, no. Pt 2, pp. 251-272. Available from: PMC.

Richmond, BG \& Whalen, M 2001, 'Forelimb function, bone curvature and phylogeny of Sivapithecus', in Phylogeny for the Neogene hominoid primates of Eurasia, eds $\mathrm{P}$ Andrews, L de Bonis \& GD Koufos, Cambridge University Press, Cambridge, pp. 326348.

Rohlf, FJ 2004, tpsDig, Stony Brook Morphometrics. Available from: http://life.bio.sunysb.edu/morph/.

Rot, C, Stern, T, Blecher, R, Friesem, B \& Zelzer, E 2014, 'A Mechanical Jack-like Mechanism Drives Spontaneous Fracture Healing in Neonatal Mice', Developmental Cell, vol. 31, no. 2, pp. 159-170. [2016/02/29].

Shackelford, LL \& Trinkaus, E 2002, 'Late Pleistocene human femoral diaphyseal curvature', American Journal of Physical Anthropology, vol. 118, no. 4, pp. 359-370.

Swartz, SM 1990, 'Curvature of the forelimb bones of anthropoid primates: Overall allometric patterns and specializations in suspensory species', American Journal of Physical Anthropology, vol. 83, no. 4, pp. 477-498.

Tardieu, C \& Trinkaus, E 1994, 'Early ontogeny of the human femoral bicondylar angle', American Journal of Physical Anthropology, vol. 95, no. 2, pp. 183-195.

Uhthoff, H \& Jaworski, Z 1978, 'Bone loss in response to long-term immobilisation', Bone \& Joint Journal, vol. 60-B, no. 3, pp. 420-429. 
331 Ullrey, DE, Sprague, JI, Becker, DE and Miller, ER, 1965, 'Growth of the swine fetus', Journal 332 of Animal Science, vol. 24, no. 3, pp.711-717.

333 Urban, JPG 1994, 'The chondrocyte: a cell under pressure', Rheumatology, vol. 33, no. 10, pp. $334901-908$.

335 West, MJ, Slomianka, L \& Gundersen, HJG 1991, 'Unbiased stereological estimation of the total 336 number of neurons in the subdivisions of the rat hippocampus using the optical fractionator', The Anatomical Record, vol. 231, no. 4, pp. 482-497. 